\title{
TaSTP13 contributes to wheat susceptibility to stripe rust possibly by increasing cytoplasmic hexose concentration
}

\author{
Baoyu Huai ${ }^{1}$, Qian Yang ${ }^{1}$, Xiaobo Wei ${ }^{2}$, Qinglin Pan ${ }^{1}$, Zhensheng Kang ${ }^{1 *}$ and Jie Liu ${ }^{2^{*}}$ (D)
}

\begin{abstract}
Background: Biotrophic fungi make intimate contact with host cells to access nutrients. Sugar is considered as the main carbon sources absorbed from host cells by pathogens. Partition, exchanges and competition for sugar at plant-pathogen interfaces are controlled by sugar transporters. Previous studies have indicated that the leaf rust resistance (Lr) gene Lr67, a natural mutation of TaSTP13 encoding a wheat sugar transport protein, confers partial resistance to all three wheat rust species and powdery mildew possibly due to weakened sugar transport activity of TaSTP13 by heterodimerization. However, one major problem that remains unresolved concerns whether TaSTP13 participates in wheat susceptibility to rust and mildew.
\end{abstract}

Results: In this study, expression of TaSTP13 was highly induced in wheat leaves challenged by Puccinia striiformis $\mathrm{f}$. sp. tritici (Pst) and certain abiotic treatments. TaSTP13 was localized in the plasma membrane and functioned as homooligomers. In addition, a functional domain for its transport activity was identified in yeast. Suppression of TaSTP13 reduced wheat susceptibility to Pst by barley stripe mosaic virus-induced gene silencing (VIGS). While overexpression of TaSTP13 promoted Arabidopsis susceptibility to powdery mildew and led to increased glucose accumulation in the leaves.

Conclusions: These results indicate that TaSTP13 is transcriptionally induced and contributes to wheat susceptibility to stripe rust, possibly by promoting cytoplasmic hexose accumulation for fungal sugar acquisition in wheat-Pst interactions.

Keywords: Wheat, Stripe rust, Sugar transporter, Susceptibility, VIGS

\section{Background}

During the Calvin cycle and gluconeogenesis processes, higher plants' source leaves convert photosynthetically fixed $\mathrm{CO}_{2}$ into sugars, such as sucrose and its cleavage products, glucose, and fructose, which represent the central units for carbon metabolism, storage, and transport [1]. These sugars not only serve as essential substrates for fundamental plant growth processes but also are major energy sources and carbon precursors [2]. Additionally, sugars are pivotal signaling molecules that directly and indirectly regulate gene expression during the plant life cycle [2]. Sugar

\footnotetext{
* Correspondence: kangzs@nwsuaf.edu.cn; liujie2003@hotmail.com

${ }^{1}$ State Key Laboratory of Crop Stress Biology for Arid Areas, College of Plant Protection, Northwest A\&F University, Yangling, China

${ }^{2}$ State Key Laboratory of Crop Stress Biology for Arid Areas, College of Life Sciences, Northwest A\&F University, Yangling, China
}

compartmentation mediated by sugar transporters is one of the major determinants for plant growth and various environmental stress responses $[1,3,4]$.

Sucrose is a major long-distance translocation form of photoassimilate that is exported from the phloem to the apoplasm of sink tissues under hydrostatic pressure, and then subsequently imported into sink cells to sustain heterotrophic metabolism and growth by specific transport proteins [5]. This export step is mediated by SWEET proteins, sucrose transporters, or monosacharide transporters (if extracellular invertases are available) $[3,5]$. These large sugar transporter families exert a finetuned regulation of sugar supply to satisfy different metabolic demands. Sugar transport proteins (STPs) are members of the monosaccharide transporters superfamily. There are 14 STPs found in Arabidopsis [1], 29 in rice [6], and 23 in barrel medic [7]. STPs are the best-

(c) The Author(s). 2020 Open Access This article is distributed under the terms of the Creative Commons Attribution 4.0 International License (http://creativecommons.org/licenses/by/4.0/), which permits unrestricted use, distribution, and 
characterized sugar transporter group in Arabidopsis, and all STP members have been identified in detail to date. All Arabidopsis STPs (AtSTPs) have been characterized as plasma membrane-localized $\mathrm{H}+$ /hexose symporters that show broad substrate specificity, with the exception of the non-functional AtSTP5 [8], AtSTP7 specific for L-arabinose and D-xylose [8], a glucosespecific transporter, AtSTP9 [9] and a galactose-specific transporter, AtSTP14 [10].

Pathogens are considered to be additional sinks that can lead to substantial changes in sugar partitioning within the plant. Uptake, exchanges, and competition for sugar at plant-pathogen interfaces are controlled by sugar transporters, and their regulation patterns are essential for determining the outcome of plant-pathogen interactions [7]. To date, there is increasing evidence that STPs play a central role in sugar translocation in pathogen-invaded plants. For example, in Arabidopsis infected with Erysiphe cichoracearum, up-regulation of AtSTP4 and the cell-wall invertase, At $\beta F R U C T 1$, correlated with the transport of glucose into sink tissues [11]. In the grapevine's response to infection by biotrophic pathogens, VvHT5, an AtSTP13 ortholog, was transcriptionally activated and enhanced sink strength during the transition from source to sink [12]. Moreover, when AtSTP13 was induced, it contributed to Arabidopsis resistance to grey mold disease by allowing living host cells to compete with Botrytis cinerea for apoplastic hexoses released by damaged tissues [13]. Additionally, AtSTP13 was phosphorylated by the BRASSINOSTEROID INSENSITIVE 1-associated receptor kinase 1 (BAK1) at threonine 485, which enhanced monosaccharide uptake activity to compete with bacteria for extracellular sugars, thus limiting the availability of extracellular sugar and depriving bacteria of an energy source, thereby restricting virulence factor delivery [14]. Clearly, apoplastic sugar control may constitute a host-defense strategy that limits a broad range of pathogens, including bacteria and fungi.

Puccinia striiformis f. sp. tritici (Pst), the causal agent of wheat stripe rust, is an obligate biotrophic fungus that acquires nutrients from host cells to survive. Sugar appears to be the main carbon source transported from host cells to pathogens [7]. However, little is known about the molecular mechanisms involved in the transfer of sugars or about the molecular responses induced in the host transport processes in response to pathogen invasion. Previous studies have found that Lr67 (a natural mutation of the sugar transporter TaSTP13) provides partial resistance to all three wheat rust species (i.e., stripe rust, leaf rust, and stem rust) and powdery mildew possibly due to a dominant-negative effect through heterodimerization with the functional transporters (TaSTP13) to reduce glucose uptake. Nevertheless, more details are still unclear. For example, whether is
TaSTP13 involved in wheat susceptibility to rust and mildew? Whether is reduce glucose uptake correlated with Lr67-mediated wheat resistance? In this study, the expression of TaSTP13 homologues was significantly induced in wheat leaves challenged by the Pst pathotype, CYR31, and abiotic treatments. Subcellular localization analysis revealed that TaSTP13 is located in the plasma membrane. A critical functional domain for its transport activity was identified by heterologus mutant complementation in Saccharomyces cerevisiae. Knockdown of TaSTP13 by the virus-induced gene-silencing (VIGS) system promoted wheat resistance to Pst. Transgenic Arabidopsis plants overexpressing TaSTP13 showed enhanced susceptibility to powdery mildew and increased glucose accumulation in the leaves. Yeast two-hybrid $(\mathrm{Y} 2 \mathrm{H})$ and bimolecular fluorescence complementation (BiFC) validated oligomerization of TaSTP13. These results suggest that TaSTP13 may contribute to wheat susceptibility to Pst by increasing fungal sugar supply.

\section{Results \\ Cloning and sequence analysis of TaSTP13}

The TaSTP13 gene was amplified from the Pst-infected Su11 cDNA sample using reverse transcription polymerase chain reaction (RT-PCR). The obtained TaSTP13 sequence was then blasted against the $T$. aestivum $\mathrm{cv}$. Chinese Spring (CS) genome sequence. Results revealed that there are three copies located on chromosomes $4 \mathrm{~A}$, $4 \mathrm{~B}$, and $4 \mathrm{D}$ in the wheat genome. The putative coding sequences of these three copies only differ in 54 nucleotides, sharing $98.62 \%$ sequence identity (Additional file 1: Figure S1). Accordingly, the amino acid sequences deduced from the three copies share $99.42 \%$ sequence identity (Additional file 2: Figure S2). And also, they are identical to the LR67sus protein form the wheat cultivar Thatcher in amino acid sequence, while they are different from the Lr67res protein form Thatcher RL6077 [15] at the two key amino acid residues: Gly144 and Val387 (Additional file 3: Figure S3). Multi-alignment of TaSTP13, LR67sus and LR67res was shown in Additional file 3: Figure S3.

The open reading frame (ORF) of TaSTP13 consists of 1545 nucleotides, which encodes a peptide of 514 amino acids with a calculated molecular weight of $56.71 \mathrm{kDa}$. The TaSTP13 protein sequence was used as a query sequence to search the most up-to-date databases. By doing so, the highest similarity STPs from other plant species were found. The protein sequence had 99.32\% identity with HvSTP13 from barley (IPK Barlex accession No: HORVU4Hr1G067450); 97.49\% identity with an STP13-like protein from Brachypodium distachyon, BdMST4 (GenBank accession No: XP_003558480.1); and 96.81\% identity with OsMST4 (an STP13-like protein) from rice (GenBank accession No: XP_015630449.1). The 
phylogenetic tree of TaSTP13 with these homologous proteins, as well as the STP family members from Arabidopsis, was constructed. These results revealed that all STP13-like proteins form a unique clade that is different from other Arabidopsis STP family members in the phylogenetic tree (Additional file 4: Figure S4), indicating that STP13 is conserved during plant evolution. In addition, TaSTP13 was most closely related to STP13-like proteins from monocotyledons, compared to those from dicotyledons (Additional file 4: Figure S4). These results suggest that TaSTP13 may also be an STP13-like protein.

\section{Expression pattern of TaSTP13 under different treatments} To determine the expression patterns of TaSTP13 in different wheat tissues, quantitative real-time PCR (qRTPCR) was performed with specific primers (Additional file 9: Table S2). Consistent with transcriptomic data from the WheatExp (https://wheat.pw.usda.gov/ WheatExp/), qRT-PCR revealed that the three copies of TaSTP13 are ubiquitously expressed in roots, leaves, flags, and spikelets (Fig. 1a). Their transcript abundance in green leaves was predominantly higher than that in other tissue (Fig. 1a). Additionally, TaSTP13 was also expressed in stems and flowers (data not shown). Transcriptome analysis of Pst-infected wheat leaves suggest that the TaSTP13 transcript level was up-regulated [16, 17]. To further confirm the increased expression characteristics, the transcript level of TaSTP13 was measured using qRT-PCR. The results illustrated that the transcript levels of all three TaSTP13 copies sharply increased by more than 25- and 10-fold at 12 and $24 \mathrm{~h}$ post-inoculation (hpi) with CYR31, respectively (Fig. 1b).

Additionally, because STPs are intensely regulated by many abiotic factors [1], the TaSTP13 response to various environmental stressors was determined. The expression levels of the three copies in wheat leaves treated with wounding, low temperature (LT), polyethyleneglycol (PEG) 6000, and $\mathrm{NaCl}$ were measured by qRT-PCR. Results revealed that TaSTP13 expression was up-regulated at $2 \mathrm{~h}$, and then decreased after the wounding treatment (Fig. 1c, Additional file 5: Figure S5A, C). During low temperature stress, transcript levels of TaSTP13 were keenly induced $2-24 \mathrm{~h}$ post treatment (hpt) and peaked at 12 hpt (Fig. 1c, Additional file 5: Figure S5A, C). TaSTP13
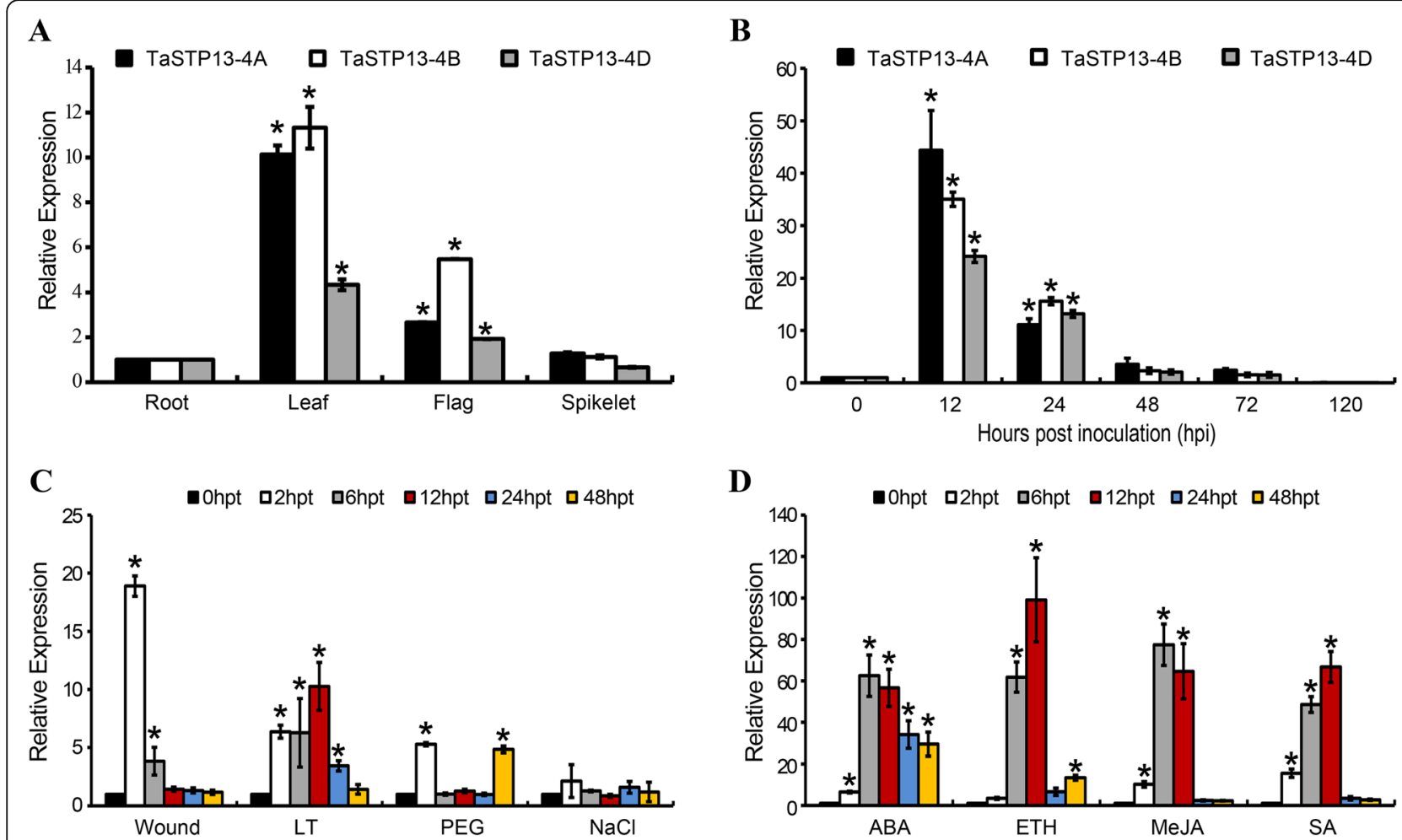

Fig. 1 Expression patterns of the three TaSTP13 copies. a Transcript profile of TaSTP13 in different wheat tissues. Samples were collected from roots, leaves, flags and spikelet. The level of TaSTP13 was defined as 1 in root tissue; levels in other tissues are presented as relative ratios. b Transcriptional changes of the three TaSTP13 copies induced by Pst infection. Wheat leaves infected with CYR31 were collected at 0, 12, 24, 48, 72, and 120 hpi. Transcript profile of TASTP13-4A in response to abiotic stress (c) and exogenous hormones (d). Wheat leaves were sampled at 0, 2, 6, 12, 24 and 48 hpt.

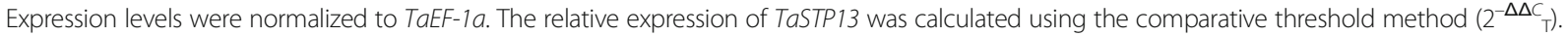
Asterisks indicate a significant difference $(P<0.01)$ according to Student's $t$-test. Bars indicate the mean \pm SD of three independent biological replicates. ABA, abscisic acid; ETH, ethylene; MeJA, methyl jasmonate; SA, salicylic acid; LT, low tempreture; PEG; polyethyleneglycol 6000 
transcripts were up-regulated at $2 \mathrm{~h}$ after PEG6000 treatment, then subsequently decreased from 6 to $24 \mathrm{hpt}$ and increased again at $48 \mathrm{hpt} \mathrm{(Fig.} \mathrm{1c,} \mathrm{Additional} \mathrm{file} \mathrm{5:} \mathrm{Figure}$ $\mathrm{S} 5 \mathrm{~A}, \mathrm{C})$. Under the $\mathrm{NaCl}$ treatment, changes were not observed in transcript abundance of TaSTP13 compared to the control (Fig. 1c, Additional file 5: Figure S5A, C).

Additionally, TaSTP13 transcript levels were assayed in wheat leaves after treatment with exogenous plant hormones abscisic acid (ABA), ethylene (ETH), methyl jasmonate (MeJA), and salicylic acid (SA). After hormone treatment, TaSTP13 expression was induced, peaking at $12 \mathrm{hpt}$ (Fig. 1d, Additional file 5: Figure S5B, D). Taken together, these results clearly indicate that the transcription of TaSTP13 responds to abiotic factors, hormone elicitors, and Pst.

\section{TaSTP13 localization in the plasma membrane}

To examine the subcellular localization of TaSTP13, TaSTP13- GFP translational fusions driven by the cauliflower mosaic virus (CaMV) 35S promoter were constructed. High fluorescence of free GFP (control) was found throughout the cell, including the nucleus (Fig. 2ab). The TaSTP13-GFP fusions were predominantly distributed at the periphery (plasma membrane) of transformed wheat leaf protoplasts. GFP fluorescence indicated plasma membrane association of TaSTP13-GFP fusion proteins (Fig. 2a). Similarly, in Nicotiana benthamiana epidermal cells, GFP fluorescence signals of TaSTP13GFP coincided with the FM4-64-labeled plasma membrane (Fig. 2b). Thus, it is likely that TaSTP13 is a plasma membrane-localized sugar transporter.

\section{Functional domain analysis of TaSTP13}

To clarify whether it is appropriate to identify the function of TaSTP13 in yeast, the TaSTP13-GFP fusion construct was transformed into a yeast ( $S$. cerevisiae) mutant, EBY.VW4000, which lacks hexose transporters and has no detectable hexose transport activity [18]. Fluorescence signals were monitored using confocal microscopy. Results revealed that TaSTP13-GFP is also localized in the plasma membrane when expressed in yeast (Fig. 3a), which is similar to what we found in plant cells. Thus, the transport properties of TaSTP13 may be determined in yeast.

The domain of TaSTP13 was analyzed using Interpro and ExPASy softwares. The results showed that a region of 18 amino acids (337-354) was possibly important for TaSTP13 (Fig. 3b). To assess whether this domain is pivotal for the transport activity of TaSTP13, mTaSTP13 (TaSTP13 lacking this region, Fig. 3b) was amplified using overlap-PCR and ligated into the pDR195 vector. The recombinant plasmid pDR195-mTaSTP13 was introduced into the EBY.VW4000 mutant. The growth of positive transformants was measured on synthetic dropout (SD) containing glucose as the sole carbon source. The results showed that there was no colony formation observed in the yeast strains harboring pDR195mTaSTP13 or empty pDR195 plasmid (Fig. 3c), whereas the complemented strain carrying pDR195-TaSTP13 could grow normally on media with glucose as the sole carbon source (Fig. 3c). These results indicate that this domain (amino acids 337-354) is functionally essential for TaSTP13.

\section{Knockdown of TaSTP13 enhances wheat resistance to Pst}

In order to identify the function of TaSTP13 in wheat leaves challenged with Pst, VIGS was utilized in this study. To make sure the three copies of TaSTP13 were co-silenced, two TaSTP13-specific fragments were selected (Additional file 1: Figure S1). As shown in Fig. 4a, BSMV-inoculated wheat plants exhibited mild chlorotic mosaic symptoms, and there was an apparent photobleaching phenotype visualized in BSMV:TaPDS-infected plants at 12 days post-inoculation (dpi) (Fig. 4a), which was used as the control for VIGS efficiency. Subsequently, the surface of fourth leaves was infected with CYR31. Reduced rust phenotypes were observed in TaSTP13-silenced wheat seedlings at $14 \mathrm{dpi}$ (Fig. 4b).

The qRT-PCR analysis revealed that the expression of the three copies of TaSTP13 was significantly suppressed, and the silencing efficiency was roughly $50 \%$ higher (Fig. 4c). To examine whether the reduced disease symptom was involved in the mycelia development of wheat leaves, fungal biomass was measured by qRTPCR. Results revealed that the TaSTP13-silenced wheat seedlings had lower fungal biomass compared to control plants (Fig. 4d). Altogether, these results indicate that down-regulation of TaSTP13 leads to decreased wheat susceptibility to stripe rust in VIGS plants.

\section{Histology of fungal growth in TaSTP13-knockdown plants}

To clarify the reduction in the disease phenotype of TaSTP13-knockdown wheat plants infected with CYR31, fungal development at different Pst infection stages was microscopically detected. At $48 \mathrm{hpi}$, hyphal branches, haustorial mother cells, and haustorias were significantly decreased in TaSTP13-silenced plants (Fig. 5a-c, j). However, the hyphal length was similar to control plants at 48 hpi (Additional file 6: Figure S6). Additionally, fungal hypha was significantly wider and had aberrant swelling structures were observed in the TaSTP13-knockdown plants (Fig. 5a, c, k-l). At 72 and 120 hpi, hyphal spreads in TaSTP13-silenced seedlings were strictly limited compared with control plants (Fig. 5d-f, g-i, m-n). These results indicate that knockdown of TaSTP13 triggered blocked hyphal development, leading to the weakened disease phenotype. 


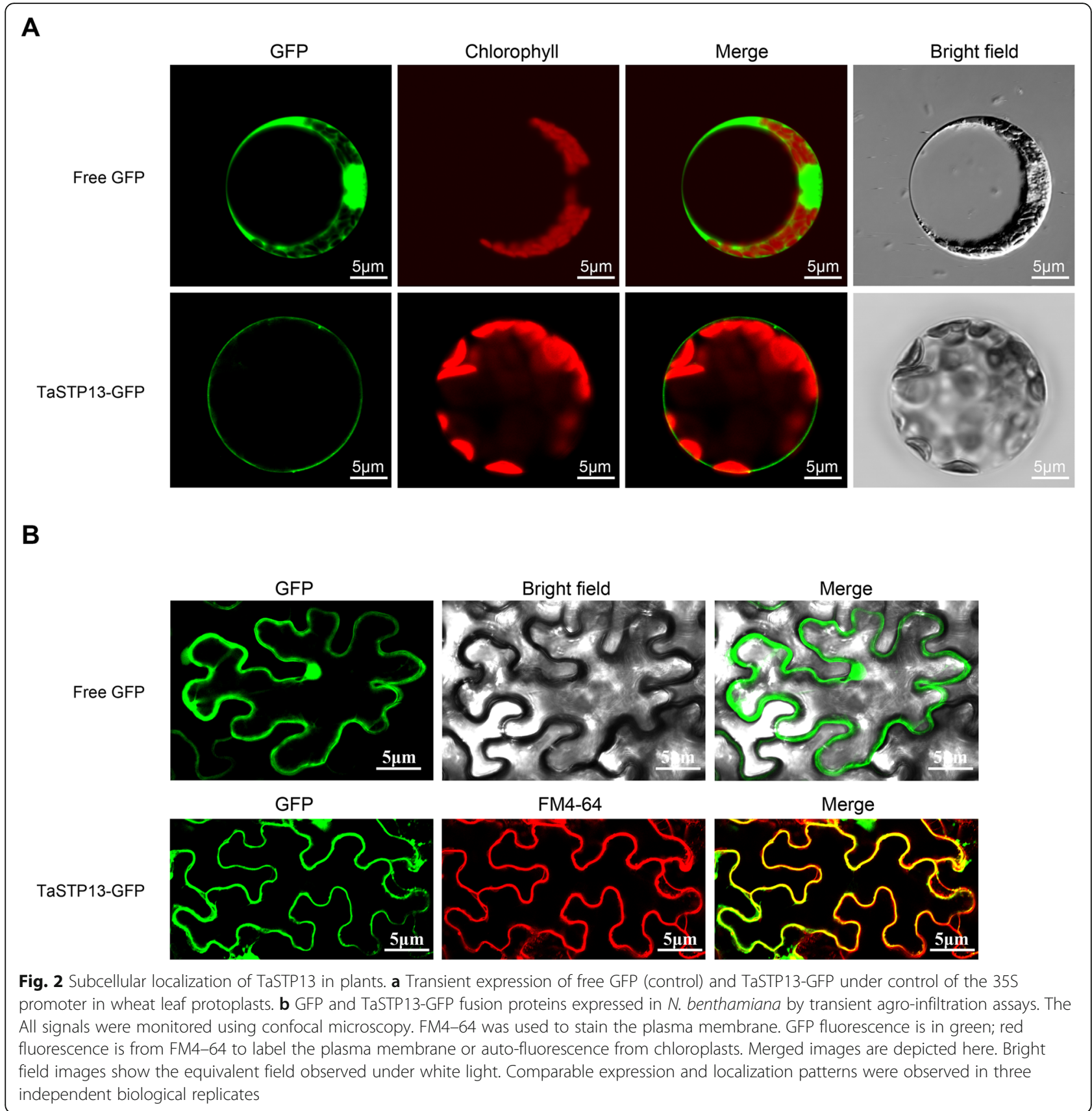

\section{Overexpression of TaSTP13 promotes Arabidopsis} susceptibility to powdery mildew

To further investigate the possible function of TaSTP13 in plant-fungal interactions, we generated several transgenic lines (TaSTP13-OE) overexpressing TaSTP13 by introducing the TaSTP13 overexpression construct into Arabidopsis. Expression of TaSTP13 in different transgenic lines in the T3 generation was confirmed using RT-PCR (Additional file 7: Figure S7). Two transgenic lines were then inoculated with tobacco powdery mildew strain Golovinomyces cichoracearum (Gc) SICAU1 [19].
We found that the TaSTP13-OE plants showed clear enhanced disease susceptibility (Fig. 6a-b). Consistent with the results at $12 \mathrm{dpi}$, TaSTP13-OE plants had significantly more conidiophores per colony than Col-0 during the early infection stage at $5 \mathrm{dpi}$ when the fungus began asexual reproduction (Fig. 6c-d). Taken together, overexpression of TaSTP13 can promote Arabidopsis susceptibility to powdery mildew.

Since plasma membrane-localized TaSTP13 can transport hexoses into yeast cells, it is reasonable to speculate that up-regulation of TaSTP13 induced by pathogens 


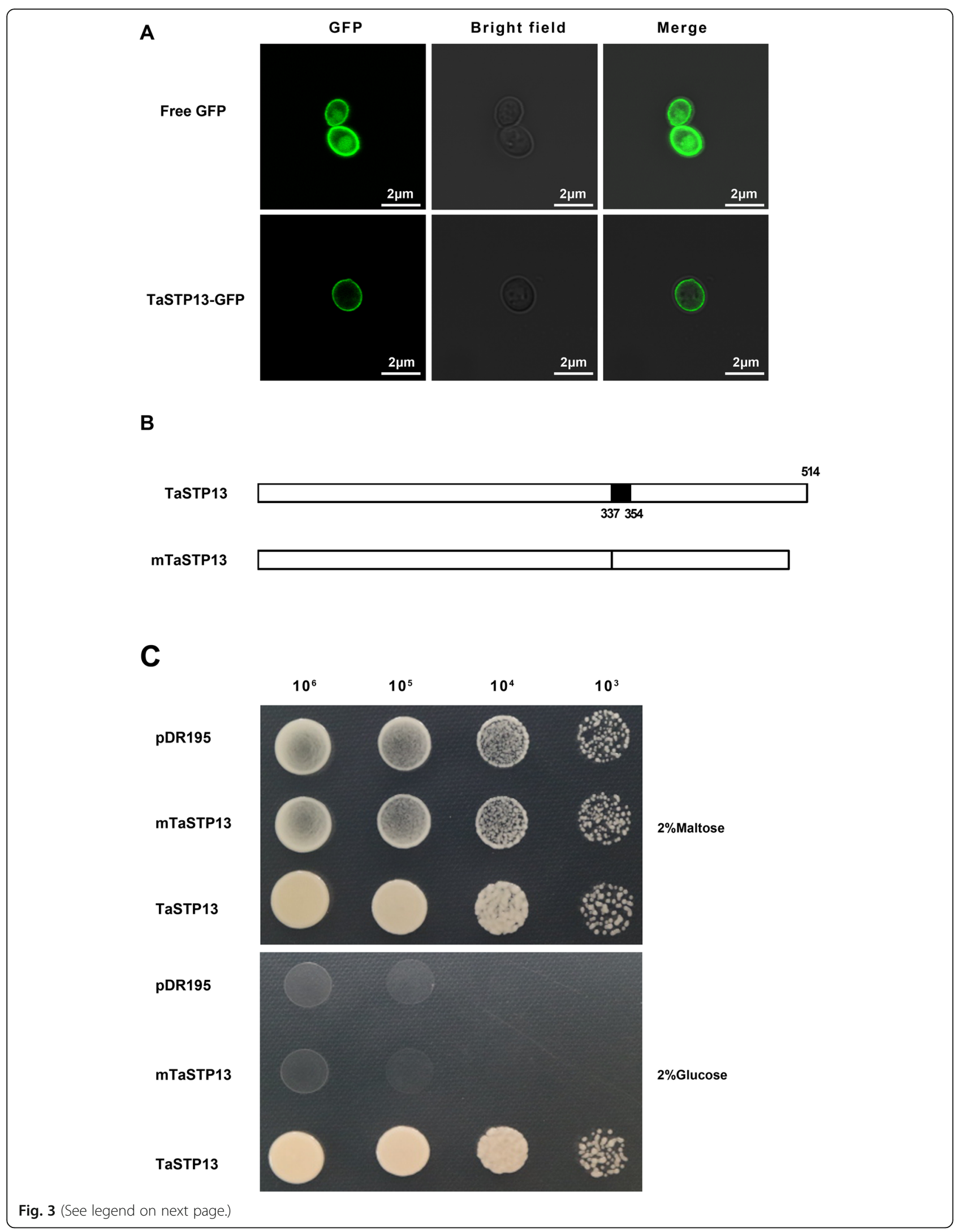


(See figure on previous page.)

Fig. 3 Expression of TaSTP13 in S. cerevisiae. a Localization of GFP and TaSTP13-GFP in EBY.WW4000. Bright field and GFP fluorescence images merged and were taken by confocal microscopy. $\mathbf{b}$ Schematic diagrams of TaSTP13 deletions. The numbers of the amino acid residues in these truncated TaSTP13 proteins are indicated. mTaSTP13, (amino acid 337 to 354) deletion. c Growth of EBY.VW4000 carrying vector pDR195, plasmid pDR195::mTaSTP13 and pDR195::TaSTP13 on maltose (control), as well as 2\% (w/v) of glucose. These experiments were repeated two times with similar results

promotes hexose accumulation in plants. To test this hypothesis, sugar concentration of leaves from these transgenic lines was then analyzed by HPLC. The results showed that glucose content in TaSTP13 overexpression plants was significantly increased compared with the wild-type control (Fig. 6e). Additionally, there was no obvious difference observed for other sugars (data not shown). These results indicate that TaSTP13 is a functional sugar transporter in plants.

\section{Oligomerization of TaSTP13}

As reported, STPs can form dimers by themselves [15]. It is therefore conceivable that TaSTP13 functions as a dimer. Thus, TaSTP13 was fused with a mutated $\mathrm{N}$ terminal half-ubiquitin protein (NubG) and C-terminal half-ubiquitin protein (Cub). The oligomerization of TaSTP13 was tested on SD media (-Trp, -Leu, -Ade, and -His) containing $\mathrm{X}-\mathrm{Gal}$ by monitoring yeast cell growth. The cells co-transformed with TaSTP13-pPR3N (-NubG) and pBT3N-TaSTP13 (-Cub) and could grow on the aforementioned media (Fig. 7a).

To evaluate the dimerization of TaSTP13 in plants, BiFC was carried out. TaSTP13 was fused with nYFP and cYFP sequences, and fusion proteins were transiently coexpressed in tobacco leaves. Strong green fluorescence signals were observed in the positive controls (TaSGT1 and TaRAR1) and in leaves co-expressing Yn:TaSTP13 and TaSTP13:Yc (Fig. 7b). In contrast, there was no fluorescence visualized in the negative controls. Thus, the results corroborate our hypothesis that TaSTP13 proteins can form homo-oligomeric complexes.

In addition, to further determine whether TaSTP13 forms hetero-oligomers with other STPs from wheat, interactions between TaSTP13 and TaSTP6, a STP conferring enhanced wheat susceptibility to stripe rust [20], were studied in yeast and tobacco plants as described above. The results showed that TaSTP13 could not interact with TaSTP6 (data not shown), indicating that TaSTP13 possibly functions as a homo-oligomer.

\section{Discussion}

Although the wheat Lr67 gene (a natural mutation of TaSTP13) has been identified as an adult plant resistance gene to all three wheat rust pathogen species and powdery mildew [15], there has been no direct evidence that has characterized the functions of TaSTP13 in wheat-rust fungi interactions. In the present study,
TaSTP13 was cloned, and its expression pattern was analyzed under a variety of treatments. In addition, the function of TaSTP13 was investigated by a BSMV-VIGS system in Pst-infected wheat and heterologous overexpression in Arabidopsis. Our results suggest that TaSTP13 may contribute to wheat susceptibility to stripe rust by increasing cytoplasmic hexose accumulation.

The previously reported STP family members were shown to be plasma membrane-localized monosaccharide $/ \mathrm{H}^{+}$symporters [7]. Results from this study revealed that TaSTP13 shares high sequence similarity with STP13-like proteins from other plants, and these proteins form a unique clade that is different from other STP family members, which exhibit highly conserved STP13 sequences throughout their evolution. Thus, it is inferred that TaSTP13 potentially encodes an STP13-like proteins. Moore et al. found that TaSTP13 could accept glucose, fructose, mannose, and galactose as substrates [15]. This broad substrate specificity is similar to AtSTP13 from Arabidopsis [21] and HvSTP13 from barley [22]. Subcellular localization analysis in wheat protoplasts, tobacco epidermal cells, and yeast showed that TaSTP13 is localized in the plasma membrane, which is consistent with previously characterized STPs [1]. Altogether, these results indicate that TaSTP13 is a broad-spectrum monosaccharide transporter localized in the plasma membrane.

In order to remain active, membrane proteins need to assemble into polymers either transiently or permanently [23]. Oligomerization is thought to play an important role in the regulation of sugar transport properties and possibly contribute to protein stability [23]. Numerous studies have reported that plant sugar transporters generally exist as homo-oligomers [15]. In this study, TaSTP13 was demonstrated to be capable of forming oligomeric structures in BiFC and $\mathrm{Y} 2 \mathrm{H}$ experiments, which is consistent with sugar transporters found in some STP and SWEET family members $[15,24]$. Recently, LR67 has been reported to restrict wheat glucose uptake through heterodimerization with functional transporters (i.e., TaSTP13) [15, 22], indicating that the homo-oligomerization of TaSTP13 is indispensable for the import of hexose from the apoplast to the cytoplasm as mediated by TaSTP13.

Sugar transport and partitioning elicited by sugar transporters is one of the most important physiological processes for plant development and response to biotic and abiotic stress $[1,3,4]$. Numerous studies have found that STPs are regulated by pathogens and participate in 


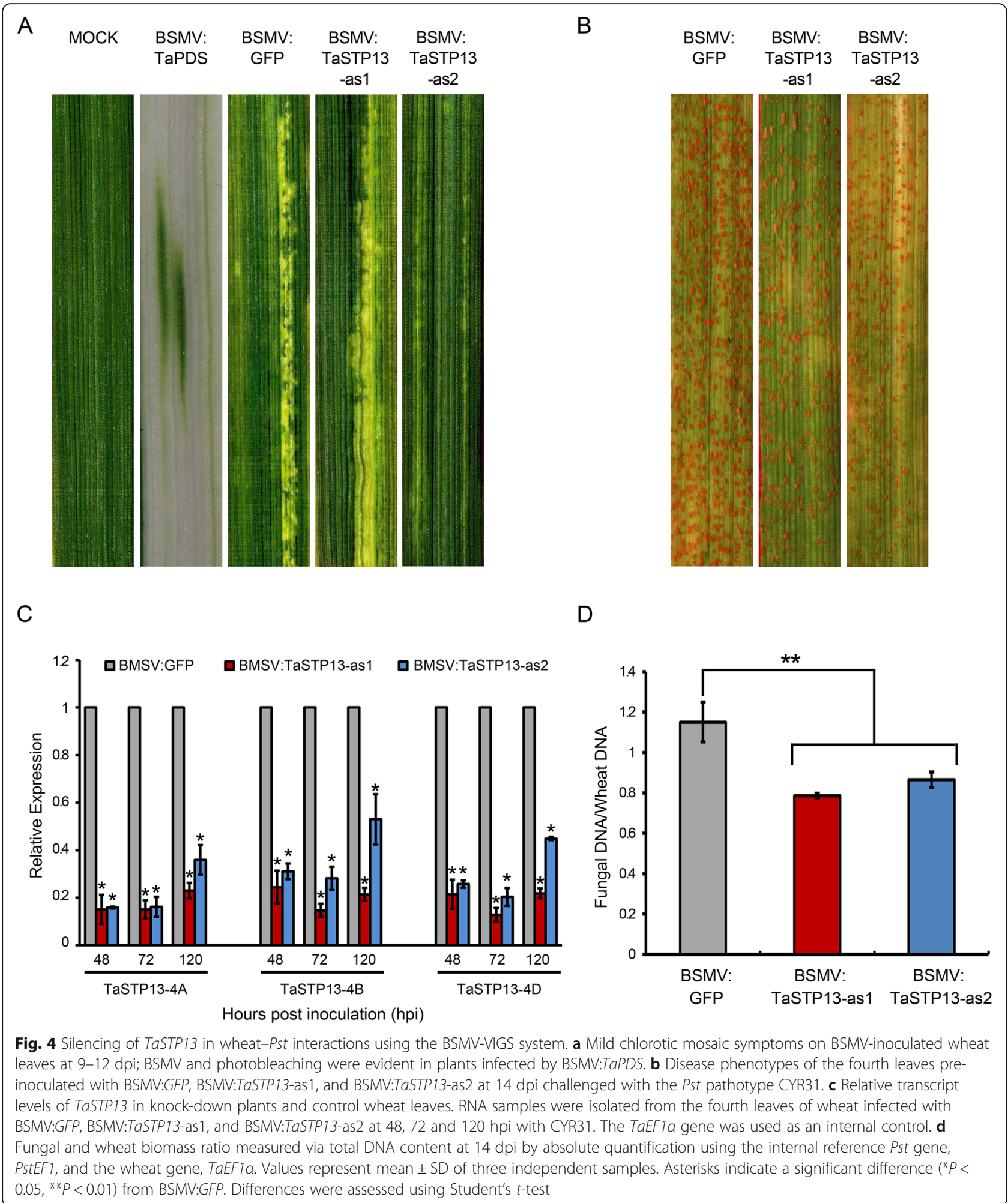

sugar transport during plant-pathogen interactions [7]. For example, AtSTP4 from Arabidopsis is transcriptionally induced after infection by E. cichoracearum and Pseudomonas syringae pv tabaci [11, 25]. Moreover, expression of AtSTP13 is up-regulated by $P$. syringae pv. tomato DC3000 and B. cinerea [13, 21]. In grapevine, the transcription level of a hexose transporter gene, $V v H T 5$, was significantly increased after powdery and 


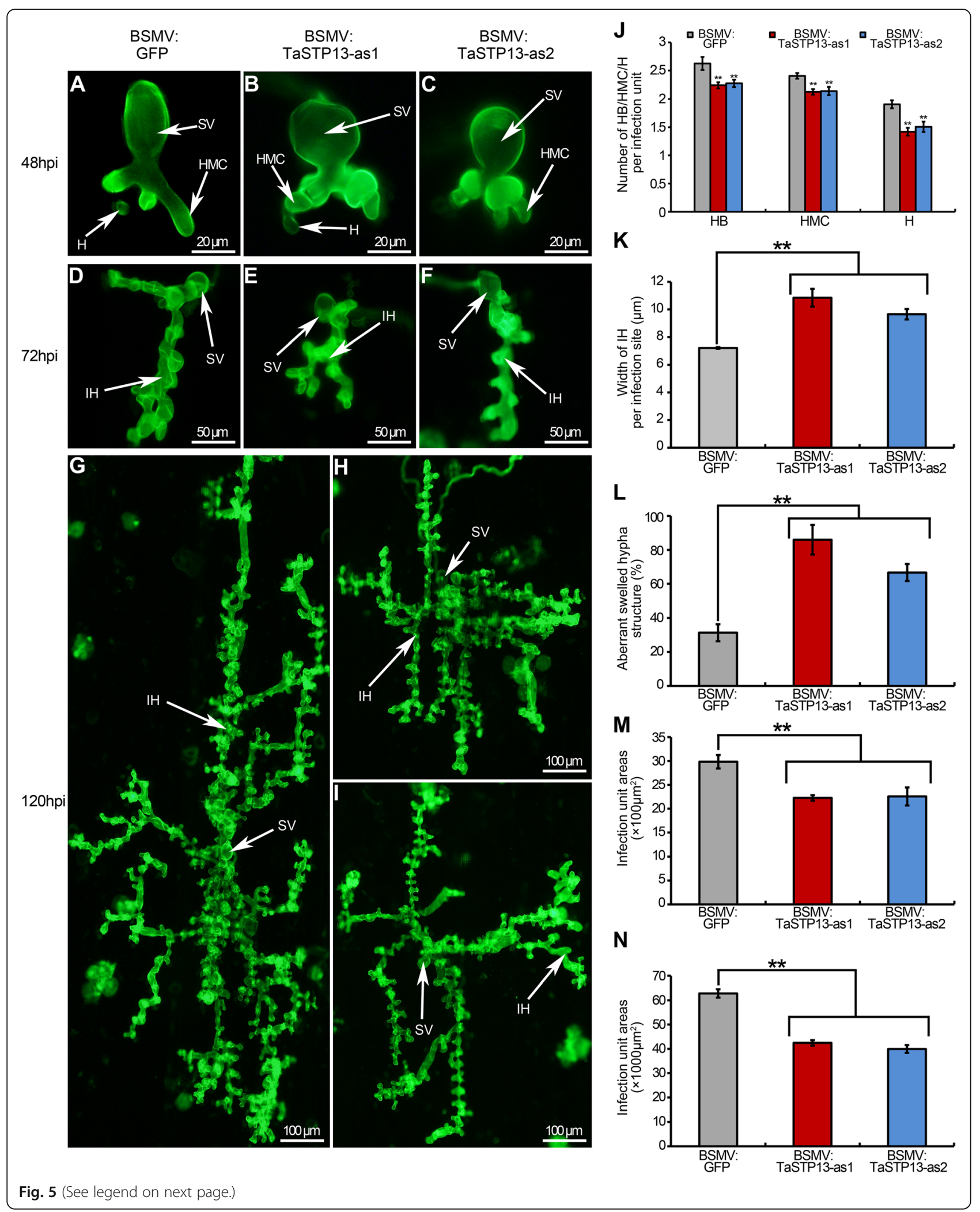


(See figure on previous page.)

Fig. 5 Histological observation of fungal growth and host response in wheat infected with BSMV:GFP and recombinant BSMV after inoculation with the Pst pathotype, CYR31. Growth of CYR31 in wheat leaves inoculated with BSMV:GFP, BSMV:TaSTP13-as1, and BSMV:TaSTP13-as2 at 48 hpi $(\mathbf{a}-\mathbf{c}), 72 \mathrm{hpi}(\mathbf{d}-\mathbf{f})$, and $120 \mathrm{hpi}(\mathbf{g}-\mathbf{i})$ was observed under a fluorescence microscope. $\mathbf{j}$ The numbers of branches of infection hyphae (HB), haustorial mother cells (HMC) and haustoria (H) per infection unit were recorded at $48 \mathrm{hpi}$. k The width of infection hyphae (IH) in TaSTP13silenced plants at $48 \mathrm{hpi}$ with the Pst pathogtype CYR31. The width of IH was approximately perpendicular to substomatal vesicle (SV) and the apex of $\mathrm{H}$. I The ratio of aberrant swelled hypha structure in wheat inoculated with BMSV constructs at $48 \mathrm{hpi}$. The aberrant swelled hypha structure was defined as hypha, of which, width was larger than the mean width of IH in BSMV:GFP-infected wheat. TaSTP13-silenced plants had a significantly reduced infection unit area at $72 \mathrm{hpi}(\mathbf{m})$ and $120 \mathrm{hpi}(\mathbf{n})$. Values represent the mean \pm SD of three independent samples with 50 infection sites each time. Asterisks indicate a significant difference $\left.{ }^{* *} P<0.01\right)$ from BSMV: GFP inoculated plants. Differences were assessed using a one-way ANOVA and Student's t-test

downy mildew infection [12]. TaSTP13 also had high expression levels in wheat during the early stages of leaf rust infection [26]. Similarly, results from this study revealed that the expression of TaSTP13 was strongly induced in Pst-infected wheat leaves, suggesting that TaSTP13 may be involved in sugar transport in wheat-rust interactions. In addition, the transcript abundance of $\operatorname{Lr} 67$ was also increased in wheat leaves infected with leaf rust [15]. The transcriptional difference seems to indicate that TaSTP13 responds differently to different rust infection.

Additionally, STPs also respond to abiotic factors, although their precise roles in regulating sugar transport during abiotic stress remain poorly understood at the molecular level [1]. In Arabidopsis, increasing AtSTP3 and AtSTP4 expression can transiently enhance response to wounding [25, 27]. AtSTP1 and AtSTP14 transcript levels displayed circadian oscillations and were found to be strongly dark-inducible $[10,28]$. Additionally, the expression of AtSTP13 has been found to be up-regulated by many abiotic factors, including multifarious elicitors, chemicals, hormones (i.e., ABA, MJ, GA biosynthesis inhibitor, PAC, and SA), and stress conditions (i.e., cold, heat, hypoxia, osmotic, oxidative, and salt) $[1,29]$. The transcriptional responses of $V v H T 5$ were induced by wounding and ABA treatment [12]. Recently, it was reported that the transcript abundance of HvSTP13 from barley was increased by salt and ABA [22]. In this study, TaSTP13 was found to be strongly induced by wounding, LT, PEG, and certain exogenous hormone treatments, which was not concerned by Moore et al. [15]. Thus, it can be speculated that sugar compartmentation mediated by TaSTP13 may be associated with stress adaptation in unfavorable conditions.

Previous studies indicated that STP13-like proteins play an important role in sugar transport during plantpathogen interactions $[21,22]$. Whether STP13-like proteins contribute to host susceptibility or resistance appears to depend on the site where pathogens acquire sugar, although these STP13-like proteins have always been found to transport sugar from the apoplast to the cytoplasm. For example, increased AtSTP13 transport activity confers Arabidopsis enhanced resistance to $B$. cinerea and $P$. syringae pv. tomato DC3000 by reducing apoplastic hexoses $[13,21]$. However, LR67 provides resistance to haustoria-forming pathogens, rust fungi, and powdery mildew by stealing sugar form host cytoplasms in wheat and decreasing the transport activity of functional transporters encoded by $\operatorname{Lr} 67$ alleles [13, 21]. In the present study, the role of TaSTP13 in wheat-Pst interactions was determined by a BSMV-VIGS system. The reduced disease symptoms, aberrant swelling structure in hyphae, and restricted colony size indicate that TaSTP13 plays a pivotal role during Pst infection in wheat. It is reported that STPs (hexose $/ \mathrm{H}^{+}$symporters) can catalyze the uptake of hexose from the apoplast to the cytoplasm [1]. Therefore we may infer that cytoplasmic sugar concentration was increased in the TaSTP13 overexpression Arabidopsis leaves. In addition, biotrophic fungi such as rust fungi or powdery mildew acquire nutrients via specialized feeding structures called haustorium by which biotrophic pathogens make intimate contact with the host cell membrane and allow for nutrient uptake $[30,31]$. Therefore we infer that upregulation of TaSTP13 may promote Arabidopsis susceptibility to powdery mildew by increasing fungal sugar supply. Compared with the previous results reported by Moore et al. [15], these results further indicate that Lr67mediated wheat rust resistance is possibly due to reduced sugar transport activity of TaSTP13. In addition, it should be pointed out that no obvious growth defects were observed in TaSTP13-silenced wheat plants, consistent with previous findings in Arabidopsis [21, 32]. One possible reason is physiologically functional redundancy among STPs. Interestingly, the functional redundancy of STPs seems not to be high in plant-pathogen interactions, possibly because contributions of different STP family members are distinct in different pathosystems.

\section{Conclusion}

Together with the results of this study and previous reports provide enough circumstantial evidence to infer that TaSTP13 plays a key role in wheat-Pst interactions and may be involved in the enhanced import of apoplastic hexoses into Pst-invaded cells. Thus, TaSTP13 may serve as a candidate gene edited to create rust-resistant wheat cultivars using the CRISPR/Cas9 system. In 


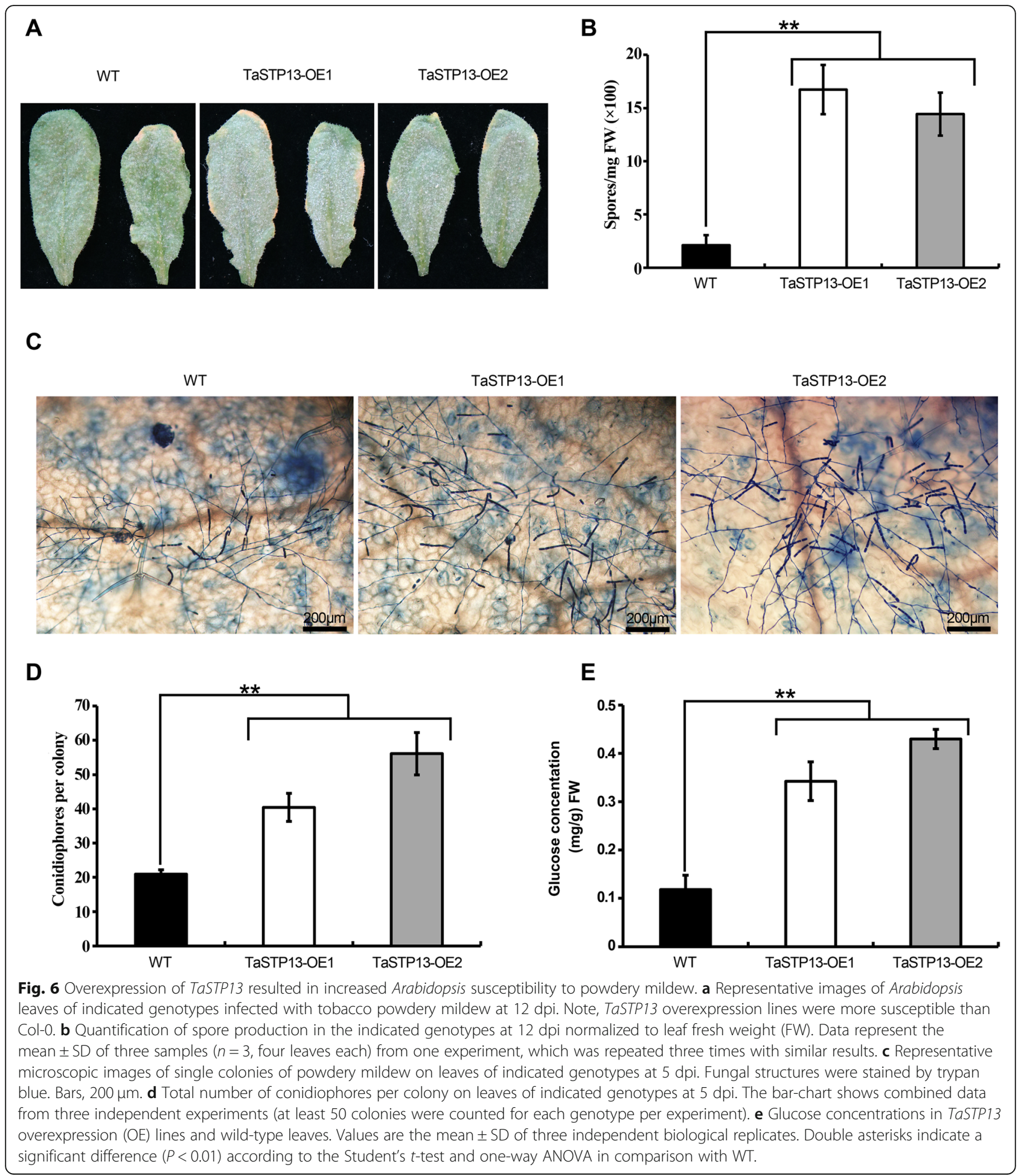

addition, the underlying mechanisms require further investigation. The identification of TaSTP13 target proteins and specific transcription factors that regulate its expression should be a goal of future research in order to complete our understanding of the exact functional mechanism.

\section{Methods}

Plant materials, inoculation, and treatments

The Arabidopsis thaliana ecotype Columbia-0, Nicotiana benthamiana, and wheat cultivar Suwon 11 (Su11) were obtained from the Prof. Zhensheng Kang's Lab (Northwest A\&F University, China) and were used in 


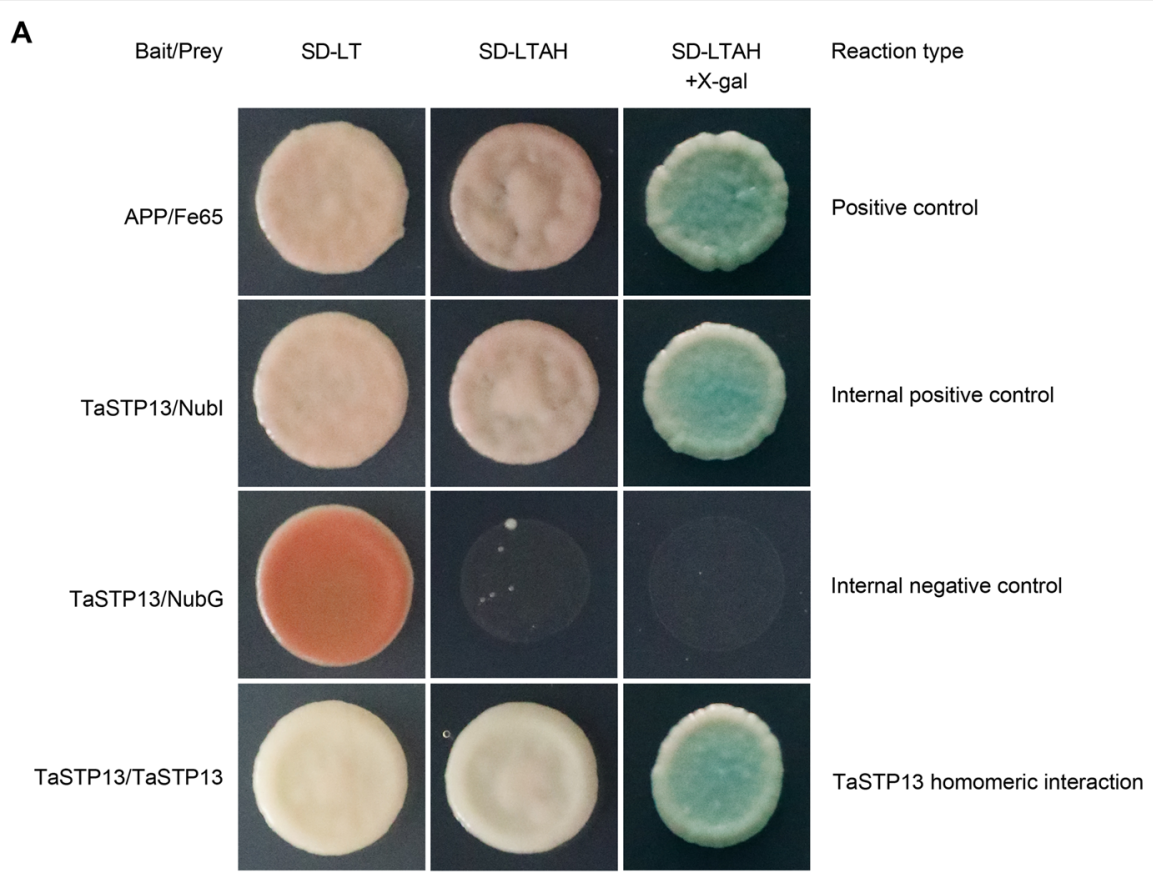

B

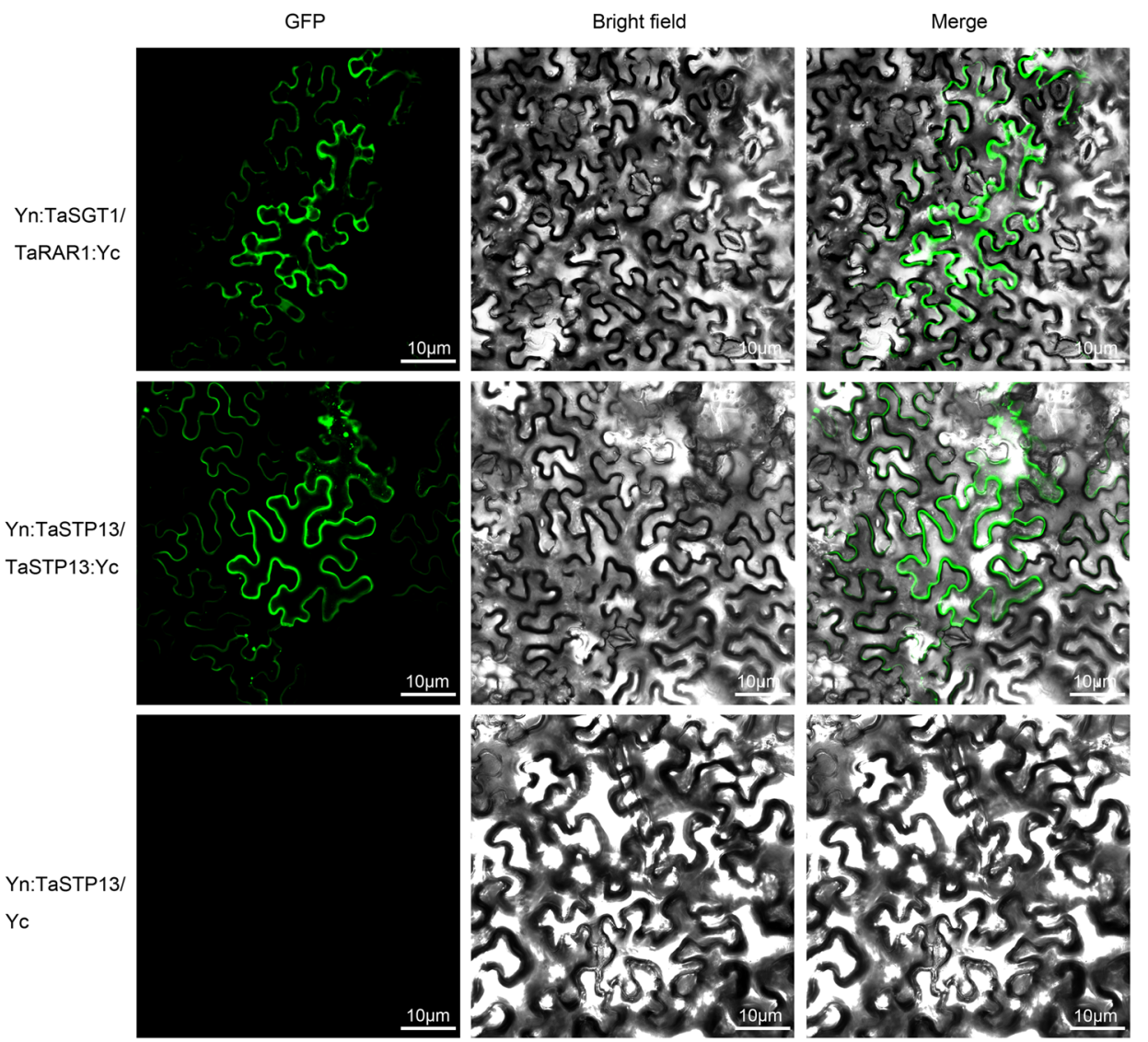

Fig. 7 (See legend on next page.) 
(See figure on previous page.)

Fig. 7 Homo-oligomerization of TaSTP13. a TaSTP13 can form homodimers within itself detected by the split-ubiquitin system using LacZ as a reporter gene. APP (amyloid A4 precursor protein) and Fe65 (amyloid beta A4 precursor protein-binding family B member 1) were used as positive control. Interactions of a TaSTP13-Cub fusion with a WT variant of Nubl (internal positive control) or mutant variant of NubG (internal negative control) and TaSTP13-Nub fusion were tested. Cells of yeast strain NMY51 harboring the indicated plasmid combinations were grown on selective media (containing $X$-gal). Positive interaction was visualized by $\beta$-galactosidase expression in drop assays. Yeast growth assays on an SD medium (- Leu, - Trp, - Ade, and - His). b BiFC assays for TaSTP13 homooligomerization. Yn:TaSGT1 + TaRAR1:Yc (positive control) is shown in the top three panels, Yn:TaSTP13 + TaSTP13:Yc is shown in the middle three panels, and Yn:TaSTP13 + Yc (negative control) is shown in the lower three panels. Agrobacterium-mediated transient expression of indicated constructs in $\mathrm{N}$. benthamiana leaves. Bright field and YFP fluorescence (in green) images were taken by confocal microscopy and merged. All assays were repeated independently at least two times with comparable results

this study. Tobacco powdery mildew isolate $G c$ SICAU1 was kindly provided by Prof. Wenming Wang's Lab (Sichuan Agricultural University, China) and was maintained on tobacco leaves at $23^{\circ} \mathrm{C}(16 \mathrm{~h}$ light, $8 \mathrm{~h}$ dark) in a growth room. Arabidopsis and $N$. benthamiana were grown following the methods described by [33].

For the tissue-specific gene expression tests, the wheat plants were grown in a growth chamber at $16^{\circ} \mathrm{C}$ for 14 days and were transferred to another growth chamber at $4{ }^{\circ} \mathrm{C}$ for 30 days. Root, leaf, flower, stem, flag, and spikelet tissues were then sampled at $1 / 2$ of complete flowering stage after about 40 days at $23^{\circ} \mathrm{C}$. The wheat plants were grown in a growth chamber, under $65 \%$ relative humidity and $200 \mu \mathrm{mol} \mathrm{m} \mathrm{m}^{-2} \mathrm{~s}^{-1}$ white light intensity for a 16-h light:8-h dark photoperiod.

Su11 and the Pst pathotype, CYR31 (virulent), were used in the wheat-Pst interactions study. To study TaSTP1 expression levels in wheat leaves infected by Pst, leaf tissues were inoculated and sampled following the methods described [33]. Time points were selected as described by [34].

For the different abiotic stressors (i.e., wounding, LT, PEG, and $\mathrm{NaCl}$ ), 14-day-old wheat seedlings were used as described [33]. For the chemical treatments, 14-dayold seedlings were sprayed with $10 \mathrm{mM} \mathrm{SA}, 1 \mathrm{mM}$ MeJA, $1 \mathrm{mM}$ ETH, or $1 \mathrm{mM}$ ABA in $0.1 \%(\mathrm{v} / \mathrm{v})$ ethanol. For the parallel mock control, wheat leaves were treated with $0.1 \%(\mathrm{v} / \mathrm{v})$ ethanol. Wheat leaves were isolated at $0,2,6$, 12 , 24, and $48 \mathrm{hpt}$ for RNA isolation.

For each experimental treatment, three independent biological replications were performed.

\section{RNA extraction and gene expression analysis}

Total RNA from wheat was extracted using a Quick RNA isolation Kit (Huayueyang Biotechnology Co., Ltd., Beijing, China). About a $2 \mu \mathrm{g}$ aliquot of total RNA was used to synthesize the first-strand cDNA with a RevertAid First Strand cDNA Synthesis Kit (Thermo Fisher Scientific, Waltham, MA, USA) with an oligo $(\mathrm{dT})_{18}$ primer. Expression levels of target genes were normalized to TaEF-1 $\alpha$ (GenBank accession No: Q03033). All qRTPCR reactions were performed in a $25-\mu \mathrm{L}$ reaction mixture containing an UltraSYBR Mixture (CWBIO Co., Ltd., Beijing, China), $10 \mathrm{pmol}$ each of the forward and reverse gene-specific primers (Additional file 8: Table $\mathrm{S} 1)$, and $2 \mu \mathrm{L}$ of diluted cDNA $(1: 20)$ that was reverse transcribed. Gene expression was quantified using a CFX Connect RT-PCR Detection System (Bio-Rad, Hercules, California, USA). The qRT-PCR analysis represented data from three biological replicates with each group containing three technical repeats. These data were analyzed by the comparative $2^{-\Delta \Delta \mathrm{Ct}}$ method [35]. Statistical significance was evaluated by Student's $t$-test.

\section{Cloning of TaSTP13 and sequence analysis}

Specific primers (Additional file 9: Table S2) from ATG to TGA were designed according to the ORFs of TaSTP13 mRNA. TaSTP13 was PCR-amplified from the Pst-infected Su11 cDNA sample using PrimeSTAR Max DNA Polymerase (TAKARA, Beijing). The PCR products were subcloned into pMD 19-T vector (Takara, Beijing, China) and sequenced. The obtained fragments were aligned with the T. aestivum cv. Chinese Spring (CS) genome using data from Ensembl Plants (http:// plants.ensembl.org) and International Wheat Genome Sequencing Consortium (https://urgi.versailles.inra.fr/ blast), and chromosomal locations were predicted.

Multiple sequence alignments were carried out using DNAMAN v6.0 (Lynnon Biosoft, USA). Polygenetic relationships were inferred using the neighbour-joining (NJ) method and bootstrap testing with 1000 replicates using MEGA v7.0 software [36]. The amino acid sequences of TaSTP13 were analyzed in ExPASy (http://www.expasy. org) to identify their physicochemical properties. The expression of TaSTP13 in different wheat tissues was analyzed in the WheatExp (https://wheat.pw.usda.gov/ WheatExp/).

\section{Constructs, primers, and strains}

DNA constructs were generated following standard molecular biology protocols or Gateway technology (Invitrogen, California, USA). More details of the DNA constructs are listed in Additional file 8: Table S1. All primers and strains are listed in Additional file 9: Table S2. 


\section{Subcellular localization}

The construct pK7FWG2-TaSTP13 was transformed into Agrobacterium tumefaciens strain GV3101 using heat shock. The positive transformants verified by PCR were cultured in LB media with spectomycin $(50 \mu \mathrm{g} / \mathrm{ml})$ and rifampicin $(50 \mu \mathrm{g} / \mathrm{ml})$ at $28^{\circ} \mathrm{C}$ in a shaking incubator at $220 \mathrm{rpm}$ for $48 \mathrm{~h}$. The bacteria were pelleted by centrifugation and resuspended in infiltration medium (10 $\mathrm{mM} \mathrm{MgCl}, 10 \mathrm{mM}$ 2-(N-morpholino) ethanesulfonic acid MES, $200 \mu \mathrm{M}$ acetosyringone, $\mathrm{pH}$ 5.7) in the dark for $3 \mathrm{~h}$ at room temperature (RT) before infiltration. The A. tumefaciens carrying construct pK7FWG2-TaSTP13 was infiltrated into tobacco ( $N$. benthamiana) leaves. Leaves were immersed in PBS consisting of $5 \mu \mathrm{M}$ FM4$64 \mathrm{~N}$-(3-triethylammoniumpropyl)-4-(6-(4-(diethyla-

mino) phenyl) hexatrienyl pyridinium dibromide (Invitrogen, USA) for $10 \mathrm{~min}$ before visualization of the plasma membrane via staining. Wheat leaf protoplast isolation and transient expression of pTF486-TaSTP13 constructs were performed as described previouly [37]. GFP fluorescence was monitored by an Olympus FV1000 confocal laser microscope (Olympus, Tokyo, Japan) with an excitation laser at $488 \mathrm{~nm}$ after transformations at $18 \mathrm{~h}$ (protoplasts) and 2 days (leaves). All assays were independently repeated three times with comparable results.

\section{Functional domain analysis of TaSTP13 in yeast}

The domain of TaSTP13 was analyzed in Interpro (https://www.ebi.ac.uk/interpro/) ExPASy (https://prosite.expasy.org/). mTaSTP13 was cloned by overlapping PCR with pairs of internal primers listed in Additional file 9: Table S2.

The empty vector pDR195 [38], as well as the constructed vectors (i.e., pDR195-TaSTP13, pDR195mTaSTP13, pDR195-GFP and pDR195-TaSTP13$G F P)$, were transformed into the hexose transport deficient S. cerevisiae mutant EBY.VW4000 [18]; these transformations were conducted using the LiAc method [39] for complementation and subcellular localization assays. Transformants were selected on $\mathrm{SD}$ media lacking uracil $\left(6.7 \mathrm{~g} \mathrm{~L}^{-1}\right.$ yeast nitrogen base, $2 \mathrm{gL}^{-1}$ amino acid (uracil) drop out mix, 2\% (w/v) maltose, and $1 \%(\mathrm{w} / \mathrm{v})$ agar). Potential clones were further verified by PCR. To determine subcellular localization in $S$. cerevisiae, yeast cells harboring pDR195-TaSTP13-GFP and pDR195-GFP (control) were visualized with a laser scanning confocal microscope, as described above. Additionally, to examine whether the predicted domain was important for the transport activity of TaSTP13, positive transformants were grown on liquid SD medium supplemented with $2 \%(\mathrm{w} / \mathrm{v})$ maltose for 1 day, and serial dilutions (i.e., $10^{3}, 10^{4}, 10^{5}$, and $10^{6}$ cells $\mathrm{mL}^{-1}$ quantified with hemacytometer) were dropped on solid media containing glucose as the sole carbon source for 3 days at $30{ }^{\circ} \mathrm{C}$.

\section{BSMV-mediated TaSTP13 gene silencing}

Two specific VIGS sequence regions from the coding sequence (260 bp, nucleotides 34-293; $219 \mathrm{bp}$, nucleotides 1264-1482, Additional file 1: Figure S1) that showed the lowest sequence similarity with other wheat genes and the highest polymorphism within STP family in a BLASTN search of the NCBI were chosen to generate their $\gamma$ RNA-based derivative plasmids as described in Additional file 8: Table S1. The BSMV:GFP vector was used as the control. Viral RNA molecules were prepared as described [40]. Plants were infected with BSMV RNA (i.e., BSMV:GFP, BSMV:TaPDS, BSMV:TaSTP13-as1, and BSMV:TaSTP13-as2) following a modified protocol $[41,42]$. At 10-12 days after virus inoculation, the fourth leaves were further treated with CYR31. Plants were then maintained at $16^{\circ} \mathrm{C}$, and fourth leaves were sampled at 48,72 , and $120 \mathrm{~h}$ post-inoculation (hpi) for histological observation and silencing efficiency. Genesilencing efficiency analysis was conducted by qRT-PCR. At 14 days after pathogen inoculation, when extensive fungal growth was visible on the leaves, infection phenotypes of Pst were photographed and collected for further analysis. Three independent inoculations were performed using 50 seedlings inoculated with each BSMV virus. Genomic DNA was extracted by the CTAB method [43]. Total genomic DNA was extracted from $P s t$-inoculated wheat leaves. The relative biomass ratio was acquired by comparing the PSEF1 [44] copy numbers with TaEF-1 $\alpha$ copy numbers.

All primers are listed in Additional file 9: Table S2.

\section{Histological observation of fungal growth}

Wheat leaf segments were fixed and bleached as described previously [33]. Wheat germ agglutinin (WGA) conjugated to Alexa Fluor 488 (Invitrogen, Carlsbad, CA, USA) was used to stain the Pst infection structures as previously described [45]. The infection sites in stained tissues were examined using an Olympus BX-51 microscope (Olympus, Tokyo, Japan) and measured with the cellSens Entry software (Olympus, Tokyo, Japan). Data of each index were obtained as described previously [20]. For the statistical analyses, standard deviations, one-way ANOVA and $t$-test were performed using SPSS software v17.0 based on three independent samples.

\section{Arabidopsis transformation and inoculation}

The pK7FWG2-TaSTP13 construct was transferred into the A. tumefaciens strain, GV3101, by heat shock for subsequent floral dipping transformation in Arabidopsis [46]. Primary transformants (T1) were selected on $1 / 2 \mathrm{MS}$ 
medium containing $50 \mu \mathrm{g} / \mathrm{ml}$ kanamycin. Selection marker resistant seedlings were verified by microscope and RT-PCR. Seedlings were then transferred into single pots filled with soil and allowed to grow until the next generation of seeds was produced in a growth chamber. Two representative homozygous lines were further analyzed. Methods of inoculation with powdery mildew and conidiophore counting were the same as those described previously [47].

\section{Extraction and determination of water-soluble carbohydrates}

The leaves of Arabidopsis for determination of watersoluble carbohydrates were collected as described previously [20]. Water-soluble carbohydrates were extracted, and high-performance liquid chromatography was performed as described [48].

\section{Split-ubiquitin analysis}

Polymerization of TaSTP13 was tested by the splitubiquitin $\mathrm{Y} 2 \mathrm{H}$ system [49]. To ensure correct expression and functionality of this system, the "bait" construct, pBT3-N-TaSTP13, was co-transformed with the prey control vector, which expresses the wild-type NubI (internal positive control) portion and the NubG (internal negative control) portion. Self-interaction of TaSTP13 was determined by co-transformation of pBT3-NTaSTP13 and the "prey" vector, pPR3N-NubG-TaSTP13. The yeast colonies were grown at $30^{\circ} \mathrm{C}$ on SD-Leu-Trp medium (SD-LT). Self-interaction of TaSTP13 was assessed by monitoring cell growth on SD-Leu-Trp-AdeHis medium (SD-LTAH) and SD-LTAH containing XGal for 4 days. Positive controls were used as described previously [20].

\section{BiFC assay}

The ORF of TaSTP13 was cloned into vectors pSPY$\mathrm{NE}(\mathrm{R}) 173$ and pSPYCE$(\mathrm{M})$ [50], respectively. Each pair of constructs was co-introduced into $N$. benthamiana leaves as described previously [24]. Two days after infiltration, YFP fluorescence was observed using a confocal laser scanning microscope (Olympus FV1000). Three independent biological repeats were carried out.

\section{Statistical analyses}

Data analysis was completed using SPSS software v17.0. Statistical significance was determined by Student's $t$-test for comparisons between two groups and one-way ANOVA (followed by LSD and Bonferroni test) for experiments with multiple treatments. In all figures, the spread of values is shown as error bars representing standard deviation of the means.

\section{Accession numbers}

Sequence data used in this study can be found in the NCBI database (http://www.ncbi.nlm.nih.gov/) with the following accession numbers: TaEF-1 $\alpha$ (Q03033), AtUBC21 (AT5G25760), TaSTP13-4A (ALL26328.1), TaSTP134B (ALL26329.1), TaSTP13-4D (All26330.1), SbSTP13 (XP_002465636.1), ZmSTP13 (NP_001310214.1), SiMST4 (XP_004985175.1), PhMST4 (XP_025795007.1), OsMST4 (XP_015630449.1), BdMST4 (XP_003558480.1), HvSTP13 (IPK Barlex accession: HORVU4Hr1G067450), VvHT5 (AAT09979.1), GmSTP13 (XP_003539275.1), NtSTP13 (XP_ 016500201.1), LeHT2 (СAB52689.1), StSTP13 (XP_ 006359910.1), AtSTP13 (NP_198006.1), AtSTP1 (NP_ 172592.1), AtSTP2 (NP_172214.5), AtSTP3 (NP_200960.2), AtSTP4 (NP_188627.1), AtSTP5 (NP_174718.1), AtSTP6 (NP_187247.1), AtSTP7 (NP_192114.1), AtSTP8 (NP_ 197997.1), AtSTP9 (NP_175449.1), AtSTP10 (NP_188628.1), AtSTP11 (NP_197718.1), AtSTP12 (NP_193879.4), and AtSTP14 (NP_177845.1).

\section{Supplementary information}

Supplementary information accompanies this paper at https://doi.org/10. 1186/s12870-020-2248-2

Additional file 1: Figure S1. Multi-alignment of the encoding sequences of three TASTP13 copies and two specific VIGS sequence regions. TASTP13-4A, TASTP13-4B, and TASTP13-4D represent TASTP13 coding regions from wheat genomes $A, B$, and $D$, respectively. Identical and similar nucleotides are shaded in black and light gray, respectively. VIGS sites are indicated by a single line.

Additional file 2: Figure S2. Multi-alignment of the TASTP13 proteins. TaSTP13-4A, TaSTP13-4B, and TaSTP13-4D represent deduced TaSTP13 proteins from the wheat genome $A, B$, and D, respectively. Identical and similar amino acid residues are shaded in black, light gray and pink, respectively.

Additional file 3: Figure S3. Multi-alignment of the TaSTP13 and LR67 protein. TaSTP13-4A, TaSTP13-4B, and TaSTP13-4D represent TaSTP13 proteins isolated from wheat genomes $A, B$, and $D$, respectively. Identical and similar nucleotide residues are shaded in black and light gray, respectively. The two amino acid residues that distinguish LR67 and TaSTP13 are blocked in a red frame.

Additional file 4: Figure S4. Phylogenetic analysis of TaSTP13. The phylogenetic tree of TaSTP13 was carried out with the MEGA7 by neighbour-joining approach. The confidence level for the groupings was estimated using 1000 bootstrap replicates. Branches are labeled with the protein names and GenBank accession numbers.

Additional file 5: Figure S5. Transcript profile of TASTP13-4B in response to abiotic stress (A) and exogenous hormones (B). Transcript profile of TASTP13-4D in response to abiotic stress (C) and exogenous hormones (D). Wheat leaves were sampled at 0, 2, 6, 12, 24 and $48 \mathrm{hpt}$. Expression levels were normalized to TaEF-1a. The relative expression of TASTP13 was calculated using the comparative threshold method $\left(2^{-\Delta \Delta C_{T}}\right)$. Significant differences are indicated with asterisks $(P<0.01)$ according to Student's t-test. Bars indicate the mean \pm SD of three independent biological replicates. ABA, abscisic acid; SA, salicylic acid; ETH, ethylene; MeJA, methyl jasmonate; LT, low tempreture; PEG; polyethyleneglycol 6000

Additional file 6: Figure S6. The length of IH in TASTP13-silenced and control plants at $48 \mathrm{hpi}$. No significant difference in the length of $\mathrm{IH}$ was observed between control and TaSTP13-silenced plants. The length of $\mathrm{HH}$ was measured from the substomatal vesicle to the apex of the longest infection hyphae. Values are represented as the mean \pm SD of three 
independent samples with 50 infection sites each. Significance was determined using one-way ANOVA.

Additional file 7: Figure S7. RT-PCR analysis of TaSTP13 expression in TaSTP13-OE and wild type plants. AtUBC21 was used as the control (bottom panel).

Additional file 8: Table S1. DNA constructs in this study.

Additional file 9: Table S2. The primers and strains used in this study.

\section{Abbreviations}

ABA: Abscisic acid; BAK1: BRASSINOSTEROID INSENSITIVE 1-associated receptor kinase 1; BiFC: Bimolecular fluorescence complementation; CaMV: Cauliflower mosaic virus; CS: Chinese Spring; Cub: C-terminal halfubiquitin protein; Dpi: Days post-inoculation; ETH: EthyleneMeJAMethyl jasmonate; FM4-64: N-(3-triethylammoniumpropyl)-4-(6-(4-(diethylamino) phenyl) hexatrienyl pyridinium dibromide; Hpi: Hours post-inoculation; Hpt: Hours post treatment; LSD: Least significant difference; LT: Low temperature; NJ: Neighbour-joining; NubG: N-terminal half-ubiquitin protein; ORF: Open reading frame; PEG: Polyethyleneglycol; Pst: Puccinia striiformis $\mathrm{f}$. sp. tritici;: qRT-PCR: Quantitative real-time PCR; RT-PCR: Reverse transcription polymerase chain reaction; SA: Salicylic acid; SD: Synthetic dropout; STPS: Sugar transport proteins; VIGS: Virus-induced gene silencing; WGA: Wheat germ agglutinin; Y2H: Yeast two-hybrid

\section{Acknowledgements}

We would like to thank Professor Eckhard Boles for providing the EBY.WW4000 mutant, State Key Laboratory of Crop Stress Biology for Arid Areas, Northwest A\&F University for use of their shared instrument platform, and Dr. Xueling Huang of State Key Laboratory of Crop Stress Biology for Arid Areas, Northwest A\&F University for assistance with Real Time PCR.

\section{Authors' contributions}

BYH, ZSK and JL conceived and designed the experiments; BYH, QY, XBW and QLP performed the experiments; BYH and $J \mathrm{~L}$ analyzed the data and wrote the paper. All authors discussed the results and commented on the manuscript. All authors have read and approved the manuscript in final version prior to submission.

\section{Funding}

This study was supported by the National Key Research and Development Program of China (grant nos. 2016YFD0100602) and the open Project of the State Key Laboratory of Crop Stress Biology for Arid Areas (CSBAA2019011). The funding agencies had no role in research design, data collection and analysis, or manuscript writing.

\section{Availability of data and materials}

All data generated in this study are included in the paper and in the supporting information files.

\section{Ethics approval and consent to participate}

Not applicable.

\section{Consent for publication}

Not applicable.

\section{Competing interests}

The authors declare that they have no competing interests.

Received: 8 November 2019 Accepted: 14 January 2020

Published online: 30 January 2020

\section{References}

1. Buttner M. The Arabidopsis sugar transporter (AtSTP) family: an update. PLANT BIOL (Stuttg). 2010;12(Suppl 1):35-41.

2. Eveland AL, Jackson DP. Sugars, signalling, and plant development. J Exp Bot. 2012;63(9):3367-77.

3. Chen LQ, Qu XQ, Hou BH, Sosso D, Osorio S, Fernie AR, Frommer WB. Sucrose efflux mediated by SWEET proteins as a key step for phloem transport. Science. 2012;335(6065):207-11.
4. Lemoine R, La Camera S, Atanassova R, Dedaldechamp F, Allario T, Pourtau $\mathrm{N}$, Bonnemain $\mathrm{J}$, Laloi M, Coutos-Thevenot P, Maurousset L, et al. Sourceto-sink transport of sugar and regulation by environmental factors. Front Plant Sci. 2013:4:272.

5. Sauer N. Molecular physiology of higher plant sucrose transporters. FEBS Lett. 2007:581(12):2309-17.

6. Deng X, An B, Zhong H, Yang J, Kong W, Li Y. A Novel Insight into Functional Divergence of the MST Gene Family in Rice Based on Comprehensive Expression Patterns. Genes (Basel). 2019;10:239.

7. Doidy J, Grace E, Kuhn C, Simon-Plas F, Casieri L, Wipf D. Sugar transporters in plants and in their interactions with fungi. Trends Plant Sci. 2012;17(7): 413-22.

8. Rottmann T, Klebl F, Schneider S, Kischka D, Ruscher D, Sauer N, Stadler R. Sugar transporter STP7 specificity for l-arabinose and d-xylose contrasts with the typical hexose transporters STP8 and STP12. Plant Physiol. 2018;176(3): 2330-50.

9. Schneidereit A, Scholz-Starke J, Buttner M. Functional characterization and expression analyses of the glucose-specific AtSTP9 monosaccharide transporter in pollen of Arabidopsis. Plant Physiol. 2003;133(1):182-90.

10. Poschet $G$, Hannich B, Buttner M. Identification and characterization of AtSTP14, a novel galactose transporter from Arabidopsis. Plant Cell Physiol. 2010;51(9):1571-80.

11. Fotopoulos V, Gilbert MJ, Pittman JK, Marvier AC, Buchanan AJ, Sauer N, Hall $J$, Williams LE. The monosaccharide transporter gene, AtSTP4, and the cellwall invertase, Atbetafruct1, are induced in Arabidopsis during infection with the fungal biotroph Erysiphe cichoracearum. Plant Physiol. 2003;132(2): $821-9$.

12. Hayes MA, Feechan A, Dry IB. Involvement of Abscisic acid in the coordinated regulation of a stress-inducible hexose transporter (VVHT5) and a Cell Wall Invertase in grapevine in response to biotrophic fungal infection. Plant Physiol. 2010;153(1):211-21.

13. Lemonnier $P$, Gaillard C, Veillet F, Verbeke J, Lemoine $R$, Coutos-Thevenot $P$, La Camera S. Expression of Arabidopsis sugar transport protein STP13 differentially affects glucose transport activity and basal resistance to Botrytis cinerea. Plant Mol Biol. 2014;85(4-5):473-84.

14. Yamada K, Saijo Y, Nakagami H, Takano Y. Regulation of sugar transporter activity for antibacterial defense in Arabidopsis. Science. 2016;354(6318): 1427-30.

15. Moore JW, Herrera-Foessel S, Lan C, Schnippenkoetter W, Ayliffe M, HuertaEspino J, Lillemo M, Viccars L, Milne R, Periyannan S, et al. A recently evolved hexose transporter variant confers resistance to multiple pathogens in wheat. Nat Genet. 2015;47(12):1494-8.

16. Dobon A, Bunting DC, Cabrera-Quio LE, Uauy C, Saunders DG. The hostpathogen interaction between wheat and yellow rust induces temporally coordinated waves of gene expression. BMC Genomics. 2016;17:380.

17. Hao Y, Wang T, Wang K, Wang X, Fu Y, Huang L, Kang Z. Transcriptome analysis provides insights into the mechanisms underlying wheat plant resistance to stripe rust at the adult plant stage. PLoS One. 2016;11(3): e150717.

18. Wieczorke R, Krampe S, Weierstall T, Freidel K, Hollenberg CP, Boles E. Concurrent knock-out of at least 20 transporter genes is required to block uptake of hexoses in Saccharomyces cerevisiae. FEBS Lett. 1999;464(3):123-8.

19. Li R, Zhang L, Yang $X$, Cao X, Wang Y, Ma X, Chandran V, Fan J, Yang H, Shang J, et al. Transcriptome analysis reveals pathways facilitating the growth of tobacco powdery mildew in Arabidopsis. Phytopathol Res. 2019; $1(1): 7$.

20. Huai B, Yang Q, Qian Y, Qian W, Kang Z, Liu J. ABA-induced sugar transporter TaSTP6 promotes wheat susceptibility to stripe rust. Plant Physiol. 2019;181(3):1328-43.

21. Nørholm MH, Nour-Eldin HH, Brodersen P, Mundy J, Halkier BA. Expression of the Arabidopsis high-affinity hexose transporter STP13 correlates with programmed cell death. FEBS Lett. 2006;580(9):2381-7.

22. Milne RJ, Dibley KE, Schnippenkoetter WH, Mascher M, Lui AC, Wang L, Lo C, Ashton AR, Ryan PR, Lagudah E. The wheat Lr67 gene of the sugar transport protein family confers multipathogen resistance in barley. Plant Physiol. 2018;179(4):1285-97.

23. Cecchetti C, Pyle E, Byrne B. Transporter oligomerisation: roles in structure and function. Biochem Soc Trans. 2019;47(1):433-40.

24. Xuan YH, Hu YB, Chen LQ, Sosso D, Ducat DC, Hou BH, Frommer WB. Functional role of oligomerization for bacterial and plant SWEET sugar transporter family. Proc Natl Acad Sci U S A. 2013;110(39):E3685-94. 
25. Truernit E, Schmid J, Epple P, Illig J, Sauer N. The sink-specific and stressregulated Arabidopsis STP4 gene: enhanced expression of a gene encoding a monosaccharide transporter by wounding, elicitors, and pathogen challenge. Plant Cell. 1996;8(12):2169-82.

26. Savadi S, Prasad P, Bhardwaj SC, Kashyap PL, Gangwar OP, Khan H, Kumar S. Temporal transcriptional changes in SAR and sugar transport-related genes during wheat and leaf rust pathogen interactions. J Plant Growth Regul. 2018:37(3):826-39.

27. Buttner M, Sauer N. Monosaccharide transporters in plants: structure, function and physiology. Biochim Biophys Acta. 2000;1465(1-2):263-74.

28. Stadler R, Buttner M, Ache P, Hedrich R, Ivashikina N, Melzer M, Shearson SM, Smith SM, Sauer N. Diurnal and light-regulated expression of AtSTP1 in guard cells of Arabidopsis. Plant Physiol. 2003;133(2):528-37.

29. Yamada K, Kanai M, Osakabe Y, Ohiraki H, Shinozaki K, Yamaguchi-Shinozaki K. Monosaccharide absorption activity of Arabidopsis roots depends on expression profiles of transporter genes under high salinity conditions. J Biol Chem. 2011:286(50):43577-86.

30. Voegele RT, Struck C, Hahn M, Mendgen K. The role of Haustoria in sugar supply during infection of broad bean by the rust fungus Uromyces fabae. Proc Natl Acad Sci U S A. 2001;98(14):8133-8.

31. Voegele RT, Wirsel S, Möll U, Lechner M, Mendgen K. Cloning and characterization of a novel invertase from the obligate biotroph Uromyces fabae and analysis of expression patterns of host and pathogen invertases in the course of infection. Mol Plant-Microbe Interact. 2006;19(6):625.

32. Schofield RA, Bi YM, Kant S, Rothstein SJ. Over-expression of STP13, a hexose transporter, improves plant growth and nitrogen use in Arabidopsis thaliana seedlings. Plant Cell Environ. 2009;32(3):271-85.

33. Liu J, Han L, Huai B, Zheng P, Chang Q, Guan T, Li D, Huang L, Kang Z. Down-regulation of a wheat alkaline/neutral invertase correlates with reduced host susceptibility to wheat stripe rust caused by Puccinia striiformis. J Exp Bot. 2015;66(22):7325-38.

34. Wang C, Huang L, Buchenauer H, Han Q, Zhang H, Kang Z. Histochemical studies on the accumulation of reactive oxygen species $\left(\mathrm{O}_{2}-\right.$ and $\left.\mathrm{H}_{2} \mathrm{O} 2\right)$ in the incompatible and compatible interaction of wheat-Puccinia striiformis f. sp. tritici. Physiol Mol Plant Pathol. 2007;71(4):230-9.

35. Pfaffl MW. A new mathematical model for relative quantification in real-time RT-PCR. Nucleic Acids Res. 2001;29(9):e45.

36. Kumar S, Stecher G, Tamura K. MEGA7: molecular evolutionary genetics analysis version 7.0 for bigger datasets. Mol Biol Evol. 2016;33(7):1870-4.

37. Ahmed SM, Liu P, Xue Q, Ji C, Qi T, Guo J, Guo J, Kang Z. TaDIR1-2, a Wheat Ortholog of Lipid Transfer Protein AtDIR1 Contributes to Negative Regulation of Wheat Resistance against Puccinia striiformis f. sp. tritici. Front Plant Sci. 2017:8:521.

38. Rentsch D, Laloi M, Rouhara I, Schmelzer E, Delrot S, Frommer WB. NTR1 encodes a high affinity oligopeptide transporter in Arabidopsis. FEBS Lett. 1995;370(3):264-8.

39. Soni R, Carmichael JP, Murray JA. Parameters affecting lithium acetatemediated transformation of Saccharomyces cerevisiae and development of a rapid and simplified procedure. Curr Genet. 1993;24(5):455-9.

40. Zhang Y, Bai Y, Wu G, Zou S, Chen Y, Gao C, Tang D. Simultaneous modification of three homoeologs of TaEDR1 by genome editing enhances powdery mildew resistance in wheat. Plant J. 2017:91(4):714-24.

41. Holzberg S, Brosio P, Gross C, Pogue GP. Barley stripe mosaic virus-induced gene silencing in a monocot plant. Plant J. 2002;30(3):315-27.

42. Scofield SR, Huang L, Brandt AS, Gill BS. Development of a virus-induced gene-silencing system for hexaploid wheat and its use in functional analysis of the Lr21-mediated leaf rust resistance pathway. Plant Physiol. 2005;138(4): 2165-73.

43. Porebski S, Bailey LG, Baum BR. Modification of a CTAB DNA extraction protocol for plants containing high polysaccharide and polyphenol components. Plant Mol Biol Rep. 1997;15(1):8-15.

44. Yin C, Chen X, Wang X, Han Q, Kang Z, Hulbert SH. Generation and analysis of expression sequence tags from haustoria of the wheat stripe rust fungus Puccinia striiformis f. sp. Tritici. BMC Genomics. 2009;10:626.

45. Ayliffe M, Devilla R, Mago R, White R, Talbot M, Pryor A, Leung H. Nonhost resistance of rice to rust pathogens. Mol Plant-Microbe Interact. 2011;24(10): 1143-55.

46. Clough SJ, Bent AF. Floral dip: a simplified method for agrobacteriummediated transformation of Arabidopsis thaliana. Plant J. 1998;16(6):735-43.
47. Xiao S, Calis O, Patrick E, Zhang G, Charoenwattana P, Muskett P, Parker JE, Turner JG. The atypical resistance gene, RPW8, recruits components of basal defence for powdery mildew resistance in Arabidopsis. Plant J. 2005;42(1):95-110.

48. Chang Q, Liu J, Wang Q, Han L, Liu J, Li M, Huang L, Yang J, Kang Z. The effect of Puccinia striiformis $\mathrm{f}$. sp. tritici on the levels of water-soluble carbohydrates and the photosynthetic rate in wheat leaves. Physiol Mol Plant Pathol. 2013;84:131-7.

49. Stagljar I, Korostensky C, Johnsson N, Te HS. A genetic system based on split-ubiquitin for the analysis of interactions between membrane proteins in vivo. Proc Natl Acad Sci U S A. 1998;95(9):5187-92.

50. Waadt R, Schmidt LK, Lohse M, Hashimoto K, Bock R, Kudla J. Multicolor bimolecular fluorescence complementation reveals simultaneous formation of alternative CBL/CIPK complexes in planta. Plant J. 2008;56(3):505-16.

\section{Publisher's Note}

Springer Nature remains neutral with regard to jurisdictional claims in published maps and institutional affiliations.

Ready to submit your research? Choose BMC and benefit from:

- fast, convenient online submission

- thorough peer review by experienced researchers in your field

- rapid publication on acceptance

- support for research data, including large and complex data types

- gold Open Access which fosters wider collaboration and increased citations

- maximum visibility for your research: over $100 \mathrm{M}$ website views per year

At BMC, research is always in progress.

Learn more biomedcentral.com/submissions 